\title{
Effectiveness of nivolumab affected by prior cetuximab use and neck dissection in Japanese patients with recurrent or metastatic head and neck cancer: results from a retrospective observational study in a real-world setting
}

\author{
Shin Kariya ${ }^{1} \cdot$ Yasushi Shimizu ${ }^{2} \cdot$ Nobuhiro Hanai $^{3} \cdot$ Ryuji Yasumatsu $^{4} \cdot$ Tomoya Yokota $^{5} \cdot$ Takashi Fujii $^{6}$. \\ Kiyoaki Tsukahara ${ }^{7} \cdot$ Masafumi Yoshida $^{8} \cdot$ Kenji Hanyu $^{9} \cdot$ Tsutomu Ueda $^{10} \cdot$ Hitoshi Hirakawa $^{11}$. Shunji Takahashi ${ }^{12}$. \\ Takeharu Ono ${ }^{13}$. Daisuke Sano ${ }^{14} \cdot$ Moriyasu Yamauchi $^{15} \cdot$ Akihito Watanabe $^{16} \cdot$ Koichi Omori $^{17}$. \\ Tomoko Yamazaki ${ }^{18}$. Nobuya Monden ${ }^{19} \cdot$ Naomi Kudo $^{20} \cdot$ Makoto Arai $^{21}$. Shuji Yonekura ${ }^{22}$. Takahiro Asakage ${ }^{23}$. \\ Akinori Fujiwara $^{24} \cdot$ Takayuki Yamada $^{25}$. Akihiro Homma ${ }^{26}$
}

Received: 22 December 2020 / Accepted: 13 March 2021 / Published online: 8 April 2021

(c) The Author(s) 2021

\begin{abstract}
Background To examine the effect of prior use of cetuximab and neck dissection on the effectiveness of nivolumab, we conducted a large-scale subgroup analysis in Japanese patients with recurrent/metastatic head and neck cancer.

Methods Data on the effectiveness of nivolumab were extracted from patient medical records. All patients were analyzed for effectiveness by prior cetuximab use. In the analyses for prior neck dissection, only patients with locally advanced disease were included.

Results Of 256 patients analyzed, 155 had received prior cetuximab. Nineteen of 50 patients with local recurrence underwent neck dissection. The objective response rate was 14.7 vs $17.2 \%(p=0.6116)$, median progression-free survival was 2.0 vs 3.1 months $(p=0.0261)$, and median overall survival was 8.4 vs 12 months $(p=0.0548)$ with vs without prior cetuximab use, respectively. The objective response rate was $23.1 \mathrm{vs} 25.9 \%(p=0.8455)$, median progression-free survival was 1.8 vs 3.0 months $(p=0.6650)$, and median overall survival was 9.1 vs 9.9 months $(p=0.5289)$ with vs without neck dissection, respectively.

Conclusions These findings support the use of nivolumab for patients with recurrent/metastatic head and neck cancer regardless of prior cetuximab use or neck dissection history.

Trial registration number UMIN-CTR (UMIN000032600), Clinicaltrials.gov (NCT03569436)
\end{abstract}

Keywords Nivolumab $\cdot$ Recurrent or metastatic head and neck cancer $\cdot$ Immune microenvironment $\cdot$ Cetuximab $\cdot$ Neck dissection

\section{Introduction}

Head and neck cancers (HNCs) are heterogeneous in nature and in 2018, affected over 887,000 patients globally [1]. In Japan, over 27,000 patients were diagnosed with HNC in 2017 [2].

Nivolumab, a fully human immunoglobulin G4 monoclonal antibody targeting programmed death-1 (PD-1),

Akihiro Homma

ak-homma@med.hokudai.ac.jp

Extended author information available on the last page of the article was approved in March 2017 in Japan for the treatment of patients with recurrent or metastatic (R/M) HNC who were treated by platinum-based chemotherapy [3] based on the survival benefits and manageable safety profile demonstrated in a global Phase III clinical trial (CheckMate 141) [4]. Currently, nivolumab has become the standard treatment for patients who have experienced recurrence within 6 months of treatment with platinum-based chemotherapy, and real-world outcomes in patients receiving nivolumab have been reported [5-7]. We also reported the largest realworld evidence for the effectiveness and safety of nivolumab in a retrospective chart review study [8]. Our results for 
effectiveness, including best overall response (BOR), progression-free survival (PFS), overall survival (OS), and safety, were consistent with data from CheckMate $141[4,8]$.

Recently, the relationship between immune microenvironment and immunotherapy in patients with $\mathrm{HNC}$ has been under focus. Cetuximab, a monoclonal antibody against the epidermal growth factor receptor (EGFR), is often used as the first line of treatment for patients with R/M HNC as a part of the EXTREME regimen $[9,10]$. Some reports have suggested that cetuximab may modulate the tumor microenvironment. Among patients with $\mathrm{HNC}$ receiving cetuximab monotherapy, immunosuppressive regulatory $\mathrm{T}$ cells were increased in those with a poor clinical outcome [11], while monocytic myeloid-derived suppressor cells (MDSCs) were significantly increased in nonresponders [12]. An ad hoc analysis of CheckMate 141 demonstrated that nivolumab had better efficacy than the investigator's choice (IC) of chemotherapy in R/M HNC regardless of prior cetuximab use [13]. Of note, only 27 Japanese patients participated in this study; therefore, the effects of prior cetuximab use in Japanese patients were not investigated. Several studies have investigated the association between the outcome of nivolumab treatment and prior cetuximab use as one of the possible prognostic factors in Japanese patients with R/M HNC [6, 14-16]. However, the sample sizes of these studies were small.

Neck dissection is performed to remove metastatic lymph nodes and/or to prevent cancer recurrence in the neck. Preclinical data suggest that neck dissection may affect the efficacy of subsequent immunotherapy as a lymph node represents a pivotal meeting point of immune cells where adaptive immunity is induced [17, 18]. In mouse models, lymph node dissection reduced the effect of immunotherapy involving PD-1 and programmed death-ligand 1 (PD-L1) inhibitory antibodies [17]. However, the effect of neck dissection on immunotherapy in humans is still unclear due to a lack of analysis in clinical or observational studies.

Here, we analyzed the effect of prior use of cetuximab and neck dissection on the effectiveness of nivolumab in Japanese patients with $\mathrm{R} / \mathrm{M} \mathrm{HNC}$ in a real-world clinical setting by using a larger sample size compared with previous reports [6, 14-16].

\section{Patients and methods}

\section{Patients}

All patients with R/M (distant sites) HNC who had been treated with nivolumab for the first time between July 1, 2017, and December 31, 2017, were included. Patients who had previously participated in a clinical trial involving antineoplastic therapy were excluded. A total of 256 patients were analyzed in this study.

\section{Study design}

This was a multicenter, noninterventional, retrospective study conducted at 23 centers in Japan. The full details of the study design have been published previously [8]. The study protocol was reviewed and approved by the Institutional Review Board/Independent Ethics Committee at each study site, and the study was conducted according to the ethical principles of the Declaration of Helsinki and the local regulations in Japan. Although informed consent was not obtained, patients were given the opportunity to decline permission for use of their clinical records for research (opt-out consent provision).

\section{Outcomes and assessments}

The primary objectives were to determine the overall effectiveness, including BOR, PFS, and OS, and to evaluate immune-related adverse events in real-world clinical practice. The objective of this subgroup analysis was to evaluate the effect of prior use of cetuximab and neck dissection on the effectiveness of nivolumab by assessing BOR, PFS, and OS.

Data from baseline until the most recent patient visit were collected from patients' medical charts using an electronic case report form. The baseline was defined as the visit before the start of nivolumab therapy. The data cutoff date was 1 year after the first treatment with nivolumab. Progression and response, primarily recorded by physicians, were measured according to investigator-assessed Response Evaluation Criteria in Solid Tumors (RECIST) version 1.1 criteria [19]. Evaluation time was not set because of the nature of this study.

\section{Statistical analyses}

Analyses for effectiveness regarding prior cetuximab use were performed in all patients. In the analyses for effectiveness based on prior neck dissection, only patients with locally advanced disease were included to evaluate the influence of regional neck dissection on the effectiveness of nivolumab. Demographic and baseline characteristics and response data were summarized using descriptive statistics (number of patients, mean, and standard deviation) for continuous effectiveness variables and frequency and percentage for categorical variables. OS and PFS were estimated and plotted using the Kaplan-Meier method and expressed as the proportion of patients who survived to a specific point in time and median duration, with the corresponding two-sided 95\% confidence intervals (CIs). Median OS and PFS were 
compared between the subgroups using the log-rank test. BOR was compared between the subgroups using the Wilcoxon rank-sum test, and objective response rate (ORR) and disease control rate (DCR) were compared between the subgroups using the Chi-square test. Statistical analyses were conducted using SAS version 9.4 (SAS Institute, Japan).

\section{Results}

\section{Baseline demographics and disease characteristics}

Overall patient baseline demographics and characteristics have been reported previously [8]. Briefly, of 256 patients, $202(78.9 \%)$ were male, and the median age was 66 years (range: $20-84$ years) (Table 1). Of all patients, 155 had received prior cetuximab treatment. Baseline disease characteristics were similar between patients with and without prior cetuximab exposure, with a few exceptions. Most patients with prior cetuximab exposure had received nivolumab treatment as a second-line (86 patients, 55.5\%) or later-line (60 patients, 38.7\%) therapy, while most patients without prior cetuximab exposure had received nivolumab treatment as first-line therapy (61 patients, 60.4\%). Among the patients with prior cetuximab exposure, 21 (13.5\%) had received cetuximab plus radiation (data not shown). Of the 50 patients with locally advanced disease, $31(62 \%)$ had not undergone neck dissection (Table 1).

\section{Effectiveness outcomes based on prior cetuximab use}

Among 256 patients, the ORRs in patients with prior cetuximab exposure and without prior cetuximab exposure were $14.7 \%$ (95\% CI, 9.2-21.8) and $17.2 \%$ (95\% CI, $10.0-26.8$ ), respectively ( $p=0.6116$; Table 2 ). The median PFS was 2.0 months (95\% CI, 1.8-2.3) for patients with prior cetuximab use and 3.1 months (95\% CI, 1.8-4.0) for patients without prior cetuximab use $(p=0.0261)$, and the estimated 1-year PFS rates were 10.2\% (95\% CI, 5.7-16.3) and 20.5\% (95\% CI, 13.1-29.0) for patients with and without prior cetuximab use, respectively (Fig. 1a).

Table 1 Baseline demographics and disease characteristics

\begin{tabular}{|c|c|c|c|c|c|c|}
\hline \multirow[t]{2}{*}{ Characteristic } & \multirow[t]{2}{*}{ All patients } & \multicolumn{2}{|c|}{ Prior cetuximab use } & \multirow{2}{*}{$\begin{array}{l}\text { All patients with } \\
\text { local recurrence }\end{array}$} & \multicolumn{2}{|c|}{ Prior neck dissection } \\
\hline & & With & Without & & With & Without \\
\hline All patients, $N(\%)$ & $256(100.0)$ & $155(100.0)$ & $101(100.0)$ & $50(100.0)$ & $19(100.0)$ & $31(100.0)$ \\
\hline \multicolumn{7}{|l|}{ Sex } \\
\hline Male, $n(\%)$ & $202(78.9)$ & $129(83.2)$ & $73(72.3)$ & $35(70.0)$ & 15 (78.9) & $20(64.5)$ \\
\hline Age, median (range), years & $66(20-84)$ & $66(20-84)$ & $66(24-80)$ & $66(29-78)$ & $63(34-72)$ & $67(29-78)$ \\
\hline$\geq 75$ years, $n(\%)$ & $24(9.4)$ & $13(8.4)$ & $11(10.9)$ & $5(10.0)$ & $0(0.0)$ & $5(16.1)$ \\
\hline \multicolumn{7}{|l|}{ ECOG PS, $n(\%)$} \\
\hline 0 & $118(46.1)$ & $67(43.2)$ & $51(50.5)$ & $24(48.0)$ & $9(47.4)$ & $15(48.4)$ \\
\hline 1 & 97 (37.9) & $64(41.3)$ & $33(32.7)$ & $17(34.0)$ & $7(36.8)$ & $10(32.3)$ \\
\hline$\geq 2^{\mathrm{a}}$ & $31(12.1)$ & $20(12.9)$ & $11(10.9)$ & $6(12)$ & $2(10.5)$ & $4(12.9)$ \\
\hline Unknown & $10(3.9)$ & $4(2.6)$ & $6(5.9)$ & $3(6.0)$ & $1(5.3)$ & $2(6.5)$ \\
\hline \multicolumn{7}{|c|}{ Nivolumab treatment line, $n(\%)$} \\
\hline 1st line & $70(27.3)$ & $9(5.8)$ & $61(60.4)$ & $15(30.0)$ & $6(31.6)$ & $9(29.0)$ \\
\hline 2nd line & $110(43.0)$ & $86(55.5)$ & $24(23.8)$ & $20(40.0)$ & $7(36.8)$ & $13(41.9)$ \\
\hline$\geq 3$ rd line & $76(29.7)$ & $60(38.7)$ & $16(15.8)$ & $15(30.0)$ & $6(31.6)$ & $9(29.0)$ \\
\hline \multicolumn{7}{|l|}{ Primary tumor site, $n(\%)$} \\
\hline Maxillary sinus $^{\mathrm{a}}$ & $14(5.5)$ & $10(6.5)$ & $4(4.0)$ & $5(10.0)$ & $1(5.3)$ & $4(12.9)$ \\
\hline Oral cavity & $56(21.9)$ & $30(19.4)$ & $26(25.7)$ & $13(26.0)$ & $10(52.6)$ & $3(9.7)$ \\
\hline Salivary gland ${ }^{\mathrm{a}}$ & $23(9.0)$ & $10(6.5)$ & $13(12.9)$ & $0(0.0)$ & $0(0.0)$ & $0(0.0)$ \\
\hline Larynx & $21(8.2)$ & $15(9.7)$ & $6(5.9)$ & $4(8.0)$ & $1(5.3)$ & $3(9.7)$ \\
\hline Nasopharynx ${ }^{a}$ & $19(7.4)$ & $7(4.5)$ & $12(11.9)$ & $3(6.0)$ & $0(0.0)$ & $3(9.7)$ \\
\hline Oropharynx & 40 (15.6) & $26(16.8)$ & $14(13.9)$ & $11(22.0)$ & $4(21.1)$ & 7 (22.6) \\
\hline Hypopharynx & $64(25.0)$ & $48(31.0)$ & $16(15.8)$ & $9(18.0)$ & $3(15.8)$ & $6(19.4)$ \\
\hline Others & $19(7.4)$ & $9(5.8)$ & $10(9.9)$ & $5(10.0)$ & $0(0.0)$ & $5(16.1)$ \\
\hline
\end{tabular}

ECOG PS Eastern Cooperative Oncology Group performance status

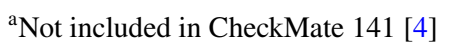


Table 2 Objective response rate in patients with and without prior cetuximab use

\begin{tabular}{lllll}
\hline Criteria & All patients & $\begin{array}{l}\text { With prior cetuxi- } \\
\text { mab }\end{array}$ & $\begin{array}{l}\text { Without } \\
\text { prior cetuxi- } \\
\text { mab }\end{array}$ & $p$ value \\
\hline $\begin{array}{c}\text { Number of } \\
\text { patients, } N\end{array}$ & $256(100.0)$ & $155(60.5)$ & $101(39.5)$ & - \\
$(\%)$ & & & & \\
BOR $^{\mathrm{a}}, n(\%)$ & $223(87.1)$ & $136(87.7)$ & $87(86.1)$ & 0.0602 \\
CR & $3(1.3)$ & $1(0.7)$ & $2(2.3)$ & \\
PR & $32(14.3)$ & $19(14.0)$ & $13(14.9)$ & \\
SD & $61(27.4)$ & $31(22.8)$ & $30(34.5)$ & \\
PD & $127(57.0)$ & $85(62.5)$ & $42(48.3)$ & \\
ORR & & & & \\
$n(\%)$ & $35(15.7)$ & $20(14.7)$ & $15(17.2)$ & 0.6116 \\
$95 \%$ CI & $(11.2-21.1)$ & $(9.2-21.8)$ & $(10.0-26.8)$ & \\
DCR $^{\mathrm{a}}$ & & & & \\
$n(\%)$ & $96(43.0)$ & $51(37.5)$ & $45(51.7)$ & 0.0364 \\
$95 \%$ CI & $(36.5-49.8)$ & $(29.4-46.2)$ & $(40.8-62.6)$ & \\
\hline
\end{tabular}

$B O R$ best overall response, $C I$ confidence interval, $C R$ complete response, $D C R$ disease control rate, $O R R$ objective response rate, $P D$ progressive disease, $P R$ partial response, RECIST Response Evaluation Criteria in Solid Tumors, $S D$ stable disease

${ }^{\mathrm{a}}$ RECIST version 1.1

The median OS was 8.4 months (95\% CI, 6.9-9.9) and 12.0 months (95\% CI, 8.3-not evaluable [NE]) for patients with and without prior cetuximab use, respectively $(p=0.0548)$, and the estimated 1 -year OS rates were $36.9 \%$ (95\% CI, 28.9-44.9) and $52.5 \%$ (95\% CI, 41.7-62.2) for patients with and without prior cetuximab use, respectively (Fig. 1b).

\section{Effectiveness outcomes based on prior neck dissection}

Among 50 patients, the ORRs in patients with prior neck dissection and without prior neck dissection were $23.1 \%$ (95\% CI, 5.0-53.8) and 25.9\% (95\% CI, 11.1-46.3), respectively ( $p=0.8455$; Table 3 ). The median PFS was 1.8 months (95\% CI, 1.2-10.0) and 3.0 months $(95 \%$ CI, 1.7-6.9) for patients with and without neck dissection, respectively $(p=0.6650)$, and the estimated 1 -year PFS rates were $14.0 \%$ (95\% CI, 2.4-35.7) and $14.9 \%$ (95\% CI, 4.7-30.4) for patients with and without neck dissection, respectively (Fig. 2a). The median OS was 9.1 months (95\% CI, 2.8-NE) and 9.9 months (95\% CI, 7.1-NE) for patients with and without neck dissection, respectively $(p=0.5289)$, and the estimated 1 -year OS rates were $36.2 \%$ (95\% CI, 13.8-59.4) and 38.5\% (95\% CI, 20.7-56.2) for patients with and without neck dissection, respectively (Fig. 2b).

\section{Discussion}

This is the first report of a large-scale retrospective study evaluating the effect of baseline characteristics such as prior use of cetuximab and neck dissection on nivolumab treatment in patients with R/M HNC in real-world clinical practice. Nivolumab showed effectiveness regardless of prior cetuximab use or neck dissection.

Cetuximab has been shown to promote differential innate and adaptive immune responses in patients with HNC treated with single-agent cetuximab [11, 12]. In these studies, cetuximab nonresponders showed an expansion of immunosuppressive regulatory $\mathrm{T}$ cells and monocytic MDSCs in the tumor microenvironment [11, 12]. Therefore, it was hypothesized that these immunosuppressive cell types would be present in patients who show disease progression after cetuximab therapy and may negatively impact a subsequent response to immunotherapy. However, the results of the current subgroup analysis were not completely in agreement with this hypothesis, and treatment effectiveness was similar irrespective of prior cetuximab use (Table 2 and Fig. 1). The results of the subgroup analysis in CheckMate 141 have been reported [13]. In patients with prior cetuximab use, the median OS $(95 \% \mathrm{CI})$ was 7.1 months (4.9-8.7) with nivolumab vs 5.1 months (4.0-6.8) with IC. In patients without prior cetuximab use, the median OS (95\% CI) was 8.2 months (5.4-9.9) with nivolumab and 4.9 months (3.1-6.5) with IC. Thus, the efficacy of nivolumab was demonstrated regardless of prior cetuximab use. In the current analysis, although the median OS for patients with prior cetuximab use was shorter than that for patients without prior cetuximab use (8.4 vs 12.0 months, $p=0.0548)$, there was no statistical significance between these two groups (Fig. 1b). Also, there was almost no difference in ORR between these two groups (14.7 vs $17.2 \%$, respectively, $p=0.6116$; Table 2 ). In contrast, the median PFS for patients with prior cetuximab use was significantly shorter than that for patients without prior cetuximab use (2.0 vs 3.1 months, respectively, $p=0.0261$; Fig. 1); however, median PFS for patients with prior cetuximab use was similar to that for the overall population (2.1 months) reported previously [8]. Taken together, these results suggest that nivolumab is efficacious regardless of prior cetuximab use. Notably, there was a significant difference in the number of lines of prior systemic cancer therapy (Table 1), although ORR, median PFS, and OS values were similar irrespective of the nivolumab line of therapy (Online Resource 1). The different numbers of prior systemic therapy may also account for the absence of impact of prior cetuximab use on the effectiveness of nivolumab observed in this study.

In this analysis, no major difference in the effectiveness of nivolumab with respect to ORR, PFS, and OS was 
Fig. 1 Overall effectiveness outcomes based on prior cetuximab use. a Progression-free survival in patients with and without prior cetuximab use. b Overall survival in patients with and without prior cetuximab use. $C I$ confidence interval, $N E$ not evaluable, $O S$ overall survival, $P F S$ progression-free survival

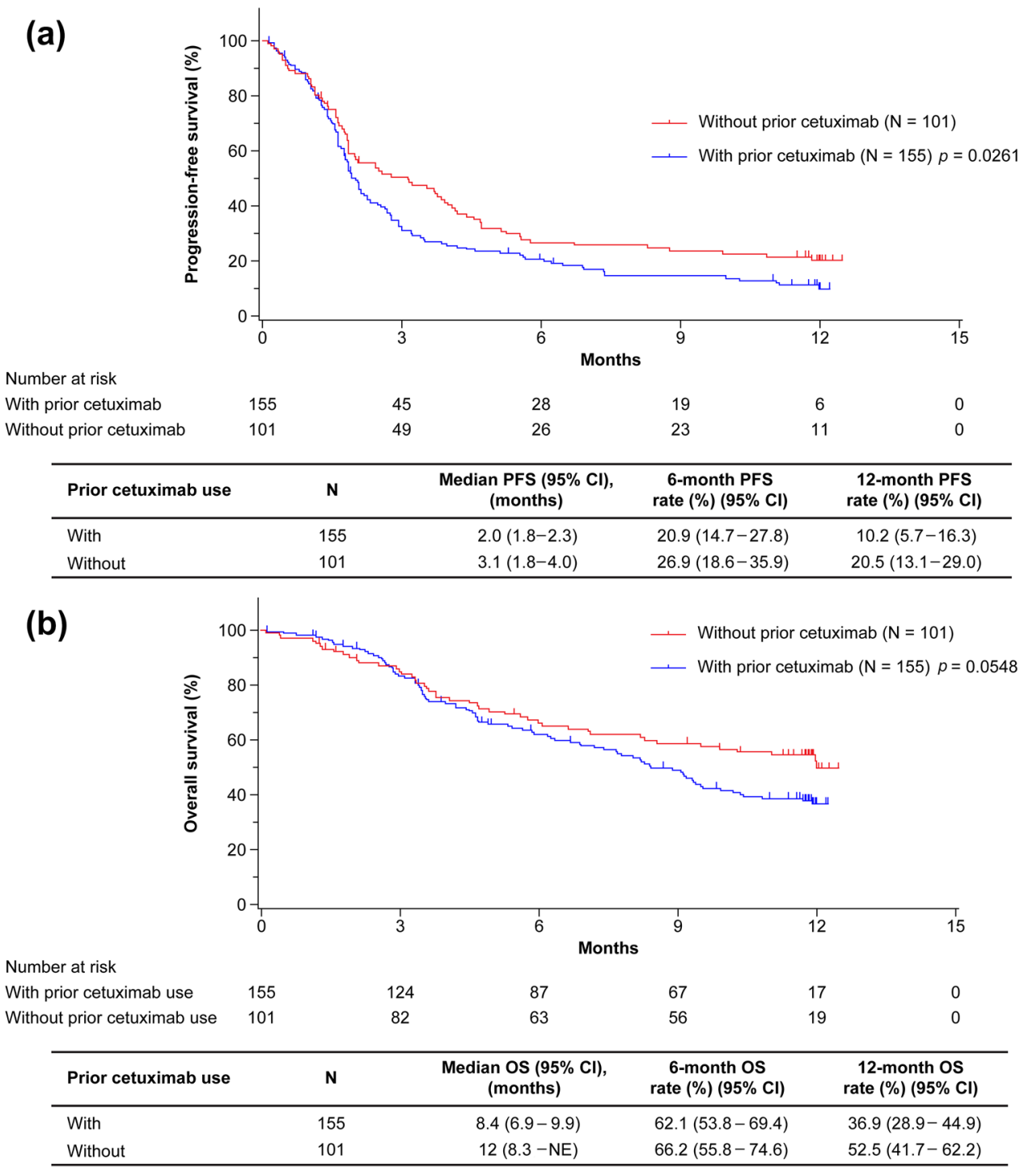

observed between patients with and without prior neck dissection (Table 3 and Fig. 2). It has been shown in mouse models that lymph node dissection reduces the effect of immunotherapy involving PD-1 and PD-L1 inhibitory antibodies [17]. In contrast, a recent study using mouse models has shown that lymph node dissection alongside established primary tumors did not affect response to immune checkpoint blockades for artificially recurrent tumors owing to the distribution of tumor-specific $\mathrm{T}$ cells from peripheral lymphatic organs [20]. To our knowledge, this is the first article reporting the effect of prior neck dissection on immunotherapy in humans. The results observed in our study are consistent with recent preclinical data [20]. A plausible reason why neck dissection did not affect the effectiveness of nivolumab in this study could be the contribution of other lymph nodes to lymphocyte migration toward the tumor site when regional lymph nodes were dissected in humans [21]. As the number of lymph nodes is considerably high in the head and neck region in humans compared with mice [22, 23], the effectiveness of nivolumab may not be impacted by neck dissection.

The inherent limitations of a retrospective, observational, real-world study design should be acknowledged. Owing to the retrospective, observational nature of the study, there was no control group. Our data were based on the assessments performed by individual physicians during their clinical practice, which could have resulted in some inconsistencies in the medical recording. We preferentially included study centers that had a greater number of patients with $\mathrm{R} / \mathrm{M} \mathrm{HNC}$, with an intent to recruit an optimal number of patients. This may have unintentionally introduced a selection bias in the study population.

In conclusion, this large-scale subgroup analysis revealed that nivolumab was efficacious regardless of prior cetuximab use or neck dissection for patients with $\mathrm{R} / \mathrm{M}$ HNC. This result supports the use of nivolumab for these 
Table 3 Objective response rate in patients with and without prior neck dissection
Fig. 2 Overall effectiveness outcomes based on prior neck dissection. a Progression-free survival in patients with and without prior neck dissection. b Overall survival in patients with and without prior neck dissection. CI confidence interval, $N E$ not evaluable, $O S$ overall survival, $P F S$ progression-free survival

\begin{tabular}{|c|c|c|c|c|}
\hline Criteria & All patients & $\begin{array}{l}\text { With prior neck dissec- } \\
\text { tion }\end{array}$ & $\begin{array}{l}\text { Without prior neck dissec- } \\
\text { tion }\end{array}$ & $p$-value \\
\hline Number of patients, $N(\%)$ & $50(100.0)$ & $19(38.0)$ & $31(62.0)$ & \\
\hline $\mathrm{BOR}^{\mathrm{a}}, n(\%)$ & $40(80.0)$ & $13(26.0)$ & $27(54.0)$ & 0.7575 \\
\hline CR & $0(0.0)$ & $0(0.0)$ & $0(0.0)$ & \\
\hline PR & $10(25.0)$ & $3(23.1)$ & $7(25.9)$ & \\
\hline SD & $7(17.5)$ & $2(15.4)$ & $5(18.5)$ & \\
\hline PD & $23(57.5)$ & $8(61.5)$ & $15(55.6)$ & \\
\hline \multicolumn{5}{|l|}{$\mathrm{ORR}^{\mathrm{a}}$} \\
\hline$n(\%)$ & $10(25.0)$ & $3(23.1)$ & $7(25.9)$ & 0.8455 \\
\hline $95 \% \mathrm{CI}$ & $(12.7-41.2)$ & $(5.0-53.8)$ & $(11.1-46.3)$ & \\
\hline \multicolumn{5}{|l|}{$\mathrm{DCR}^{\mathrm{a}}$} \\
\hline$n(\%)$ & $17(42.5)$ & $5(38.5)$ & $12(44.4)$ & 0.7200 \\
\hline $95 \% \mathrm{CI}$ & $(27.0-59.1)$ & $(13.9-68.4)$ & $(25.5-64.7)$ & \\
\hline
\end{tabular}

$B O R$ best overall response, $C I$ confidence interval, $C R$ complete response, $D C R$ disease control rate, $O R R$ objective response rate, $P D$ progressive disease, $P R$ partial response, $R E C I S T$ Response Evaluation Criteria in Solid Tumors, $S D$ stable disease

${ }^{a}$ RECIST version 1.1

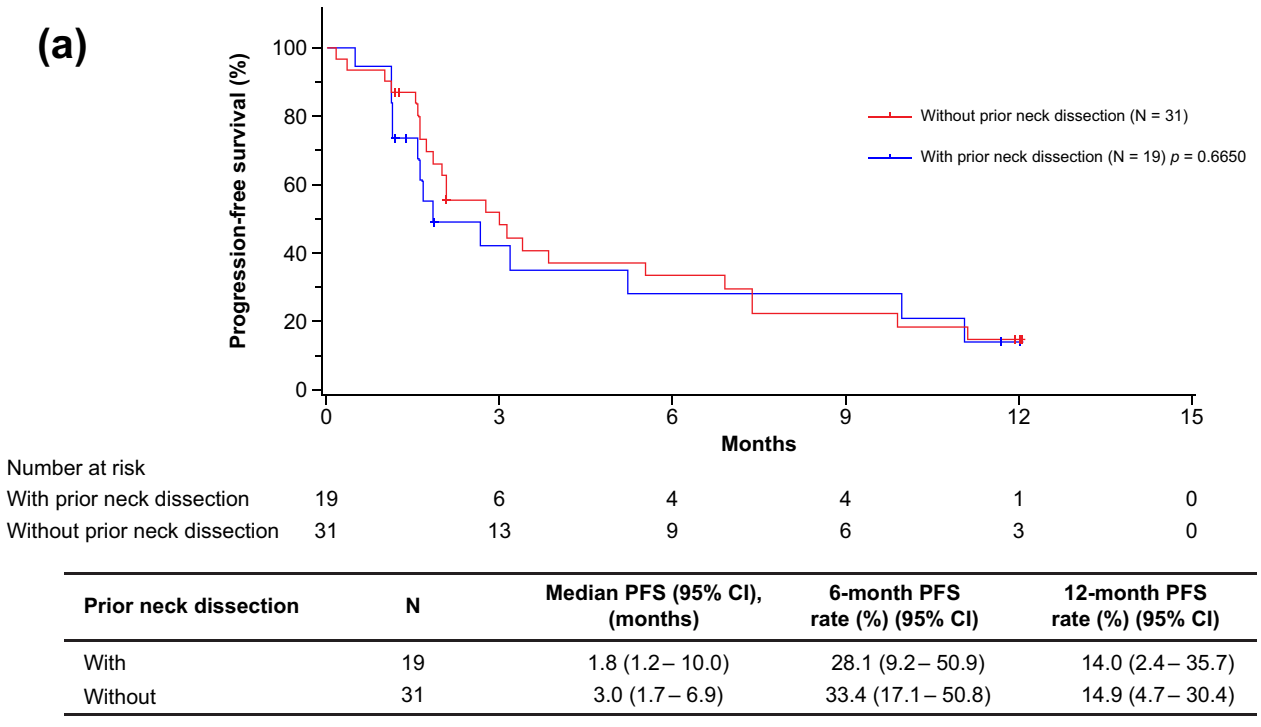

(b)

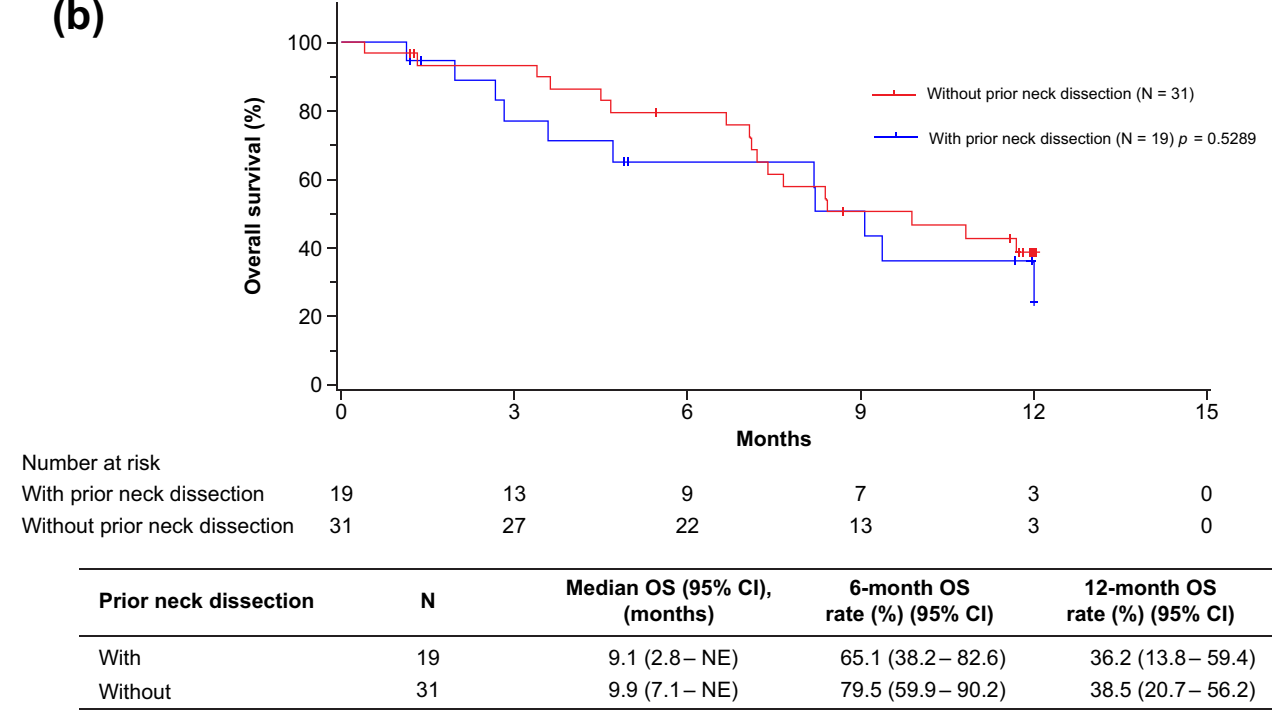


patients regardless of prior cetuximab use or neck dissection history.

Supplementary Information The online version contains supplementary material available at https://doi.org/10.1007/s10147-021-01900-4.

Acknowledgments Medical writing support was provided by Dr. Jidnyasa Mulekar, PhD, of Cactus Life Sciences (part of Cactus Communications, Mumbai, India) and was funded by Bristol-Myers Squibb and Ono Pharmaceutical Co., Ltd.

Author contributions AF, TY, and AH contributed to the study conception and design. All authors contributed to the acquisition, analysis, or interpretation of data. The first draft of the manuscript was written by $\mathrm{NH}, \mathrm{TY}$, and $\mathrm{AH}$, and all authors critically reviewed previous versions of the manuscript for important intellectual content. All authors read and gave final approval of the manuscript to be published and take responsibility for the integrity of the work as a whole.

Funding This work was funded by Bristol-Myers Squibb and Ono Pharmaceutical Co., Ltd. Bristol-Myers Squibb and Ono Pharmaceutical Co., Ltd. participated in the study design; research; data collection; data analysis and interpretation; and writing, reviewing, and approval of the publication.

\section{Declarations}

Conflict of interest N. Hanai, K. Omori, and A. Homma report grants from Bristol-Myers Squibb K.K. and Ono Pharmaceutical Co., Ltd. during the conduct of the study. S. Takahashi reports grants and personal fees from AstraZeneca, Bayer, Bristol-Myers Squibb K.K., Chugai Pharma, MSD, and Ono Pharmaceutical Co., Ltd. during the conduct of the study. K. Tsukahara reports personal fees from Bristol-Myers Squibb K.K. and Ono Pharmaceutical Co., Ltd. during the conduct of the study. T. Ueda reports grant from Ono Pharmaceutical Co., Ltd. during the conduct of the study. T. Yamazaki reports grants from AstraZeneca K.K., Bristol-Myers Squibb K.K. and Ono Pharmaceutical Co., Ltd. during the conduct of the study. R. Yasumatsu reports grants from Ono Pharmaceutical Co., Ltd. during the conduct of the study. T. Yokota reports advisory role for Merck Biopharma and personal fees from Bristol-Myers Squibb K.K, Merck Biopharma, and Ono Pharmaceutical Co., Ltd. during the conduct of the study. A. Fujiwara is an employee of Ono Pharmaceutical Co., Ltd. T. Yamada is an employee of Bristol-Myers Squibb K.K. M. Arai, T. Asakage, T. Fujii, K. Hanyu, H. Hirakawa, S. Kariya, N. Kudo, N. Monden, T. Ono, D. Sano, Y. Shimizu, A. Watanabe, M. Yamauchi, S. Yonekura, and M. Yoshida have nothing to disclose during the conduct of the study.

Open Access This article is licensed under a Creative Commons Attribution 4.0 International License, which permits use, sharing, adaptation, distribution and reproduction in any medium or format, as long as you give appropriate credit to the original author(s) and the source, provide a link to the Creative Commons licence, and indicate if changes were made. The images or other third party material in this article are included in the article's Creative Commons licence, unless indicated otherwise in a credit line to the material. If material is not included in the article's Creative Commons licence and your intended use is not permitted by statutory regulation or exceeds the permitted use, you will need to obtain permission directly from the copyright holder. To view a copy of this licence, visit http://creativecommons. org/licenses/by/4.0/.

\section{References}

1. Bray F, Ferlay J, Soerjomataram I et al (2018) Global cancer statistics 2018: GLOBOCAN estimates of incidence and mortality worldwide for 36 cancers in 185 countries. CA Cancer J Clin 68:394-424

2. Vital Statistics. National Cancer Center Japan. Available via https://ganjoho.jp/reg_stat/statistics/stat/summary.html. Accessed 9 Jun 2020

3. ONO receives approval for OPDIVO ${ }^{\circledR}$ (nivolumab) intravenous infusion for treatment of recurrent or metastatic head and neck cancer as a partial change in approved items of manufacturing and marketing approval in Japan. Ono Pharmaceutical Co., Ltd. Available via https://www.ono-pharma.com/sites/default/files/en/ news/press/sm_cn170324.pdf. Accessed 20 Apr 2020

4. Ferris RL, Blumenschein G Jr, Fayette J et al (2016) Nivolumab for recurrent squamous-cell carcinoma of the head and neck. N Engl J Med 375:1856-1867

5. Okamoto I, Sato H, Kondo T et al (2019) Efficacy and safety of nivolumab in 100 patients with recurrent or metastatic head and neck cancer-a retrospective multicentre study. Acta Otolaryngol 139:918-925

6. Hori R, Shinohara S, Kojima T et al (2019) Real-world outcomes and prognostic factors in patients receiving nivolumab therapy for recurrent or metastatic head and neck carcinoma. Cancers (Basel) 11:1317

7. Sato Y, Fukuda N, Wang X et al (2020) Efficacy of nivolumab for head and neck cancer patients with primary sites and histological subtypes excluded from the CheckMate-141 trial. Cancer Manag Res 12:4161-4168

8. Hanai N, Shimizu Y, Kariya S et al (2021) Effectiveness and safety of nivolumab in patients with head and neck cancer in Japanese real-world clinical practice: a multicenter retrospective clinical study. Int J Clin Oncol 26:494-506

9. Graham J, Muhsin M, Kirkpatrick P (2004) Cetuximab. Nat Rev Drug Discov 3:549-550

10. Vermorken JB, Mesia R, Rivera F et al (2008) Platinum-based chemotherapy plus cetuximab in head and neck cancer. N Engl J Med 359:1116-1127

11. Jie HB, Schuler PJ, Lee SC et al (2015) CTLA-4(+) regulatory T cells increased in cetuximab-treated head and neck cancer patients suppress NK cell cytotoxicity and correlate with poor prognosis. Cancer Res 75:2200-2210

12. Li J, Srivastava RM, Ettyreddy A et al (2015) Cetuximab ameliorates suppressive phenotypes of myeloid antigen presenting cells in head and neck cancer patients. J Immunother Cancer 3:54

13. Ferris RL, Licitra L, Fayette J et al (2019) Nivolumab in patients with recurrent or metastatic squamous cell carcinoma of the head and neck: efficacy and safety in CheckMate 141 by prior cetuximab use. Clin Cancer Res 25:5221-5230

14. Nishikawa D, Suzuki H, Koide Y et al (2018) Prognostic markers in head and neck cancer patients treated with nivolumab. Cancers (Basel) 10:466

15. Suzuki C, Kiyota N, Imamura Y et al (2020) Effect of tumor burden and growth rate on treatment outcomes of nivolumab in head and neck cancer. Int J Clin Oncol 25:1270-1277

16. Ueki Y, Takahashi T, Ota $\mathrm{H}$ et al (2020) Predicting the treatment outcome of nivolumab in recurrent or metastatic head and neck squamous cell carcinoma: prognostic value of combined performance status and modified Glasgow prognostic score. Eur Arch Otorhinolaryngol 277:2341-2347

17. Fransen MF, Schoonderwoerd M, Knopf P et al (2018) Tumordraining lymph nodes are pivotal in PD-1/PD-L1 checkpoint therapy. JCI Insight 3:e124507 
18. Gasteiger G, Ataide M, Kastenmüller W (2016) Lymph node-an organ for T-cell activation and pathogen defense. Immunol Rev 271:200-220

19. Eisenhauer EA, Therasse P, Bogaerts J et al (2009) New response evaluation criteria in solid tumours: revised RECIST guideline (version 1.1). Eur J Cancer 45:228-247

20. Zhao X, Kassaye B, Wangmo D et al (2020) Chemotherapy but not the tumor-draining lymph nodes determine the immunotherapy response in secondary tumors. iScience 23:101056

21. Ellis RJ, Wernick G, Zabriskie JB et al (1975) Immunologic competence of regional lymph nodes in patients with breast cancer. Cancer 35:655-659
22. Koroulakis A, Agarwal M (Updated Nov 30, 2019). Anatomy, head and neck, lymph nodes. In: StatPearls. Treasure Island (FL): StatPearls Publishing; Jan 2020. Available via https://www.ncbi. nlm.nih.gov/books/NBK513317/. Accessed 9 Jun 2020

23. Van den Broeck W, Derore A, Simoens P (2006) Anatomy and nomenclature of murine lymph nodes: descriptive study and nomenclatory standardization in BALB/cAnNCrl mice. J Immunol Methods 312:12-19

Publisher's Note Springer Nature remains neutral with regard to jurisdictional claims in published maps and institutional affiliations.

\section{Authors and Affiliations}

\section{Shin Kariya ${ }^{1} \cdot$ Yasushi Shimizu $^{2} \cdot$ Nobuhiro Hanai $^{3} \cdot$ Ryuji Yasumatsu $^{4} \cdot$ Tomoya Yokota $^{5} \cdot$ Takashi Fujii $^{6}$. Kiyoaki Tsukahara ${ }^{7} \cdot$ Masafumi Yoshida $^{8} \cdot$ Kenji Hanyu $^{9} \cdot$ Tsutomu Ueda $^{10} \cdot$ Hitoshi Hirakawa $^{11}$. Shunji Takahashi ${ }^{12}$. Takeharu Ono ${ }^{13}$. Daisuke Sano ${ }^{14} \cdot$ Moriyasu Yamauchi $^{15} \cdot$ Akihito Watanabe $^{16} \cdot$ Koichi Omori $^{17}$. Tomoko Yamazaki ${ }^{18}$. Nobuya Monden ${ }^{19} \cdot$ Naomi Kudo $^{20} \cdot$ Makoto Arai $^{21}$. Shuji Yonekura ${ }^{22}$. Takahiro Asakage ${ }^{23}$. Akinori Fujiwara ${ }^{24}$ - Takayuki Yamada ${ }^{25}$. Akihiro Homma ${ }^{26}$}

1 Department of Otolaryngology, Head and Neck Surgery, Okayama University Hospital, Okayama, Japan

2 Department of Medical Oncology, Faculty of Medicine and Graduate School of Medicine, Hokkaido University, Sapporo, Japan

3 Department of Head and Neck Surgery, Aichi Cancer Center Hospital, Nagoya, Japan

4 Department of Otolaryngology, Graduate School of Medical Sciences, Kyushu University, Fukuoka, Japan

5 Division of Gastrointestinal Oncology, Shizuoka Cancer Center, Nagaizumi, Japan

6 Department of Head and Neck Surgery, Osaka International Cancer Institute, Osaka, Japan

7 Department of Otorhinolaryngology, Head and Neck Surgery, Tokyo Medical University, Tokyo, Japan

8 Department of Otorhinolaryngology and Head and Neck Surgery, The University of Tokyo Hospital, Tokyo, Japan

9 Head and Neck Oncology Center, International University of Health and Welfare, Mita Hospital, Tokyo, Japan

10 Department of Otorhinolaryngology, Head and Neck Surgery, Hiroshima University Hospital, Hiroshima, Japan

11 Department of Otorhinolaryngology, Head and Neck Surgery, University of the Ryukyu Hospital, Nishihara, Japan

12 Department of Medical Oncology, The Cancer Institute Hospital of Japanese Foundation for Cancer Research, Tokyo, Japan

13 Department of Otolaryngology, Head and Neck Surgery, Kurume University Hospital, Kurume, Japan
14 Department of Otolaryngology, Head and Neck Surgery, Yokohama City University Hospital, Yokohama, Japan

15 Department of Otolaryngology, Head and Neck Surgery, Saga University Hospital, Saga, Japan

16 Department of Otolaryngology, Head and Neck Surgery, Keiyukai Sapporo Hospital, Sapporo, Japan

17 Department of Otolaryngology, Head and Neck Surgery, Kyoto University Hospital, Kyoto, Japan

18 Division of Head and Neck Cancer Oncology, Miyagi Cancer Center, Sendai, Japan

19 Department of Head and Neck Surgery, National Hospital Organization Shikoku Cancer Center, Matsuyama, Japan

20 Department of Otorhinolaryngology, Hirosaki University Graduate School of Medicine, Hirosaki, Japan

21 Department of Medical Oncology, Chiba University Hospital, Chiba, Japan

22 Department of Otorhinolaryngology, Head and Neck Surgery, Chiba University Hospital, Chiba, Japan

23 Department of Head and Neck Surgery, Tokyo Medical and Dental University Medical Hospital, Tokyo, Japan

24 Medical Affairs, Ono Pharmaceutical Co., Ltd., Osaka, Japan

25 Japan Medical and Development, Bristol-Myers Squibb K.K., Tokyo, Japan

26 Department of Otolaryngology, Head and Neck Surgery, Faculty of Medicine and Graduate School of Medicine, Hokkaido University, Kita15 Nishi7, Kita-Ku, Sapporo, Hokkaido 060-8638, Japan 